\title{
大比例尺地形图中小型无人机的实践
}

\section{Practice of Small and Medium UAV in Large Scale Topographic Map \\ 魏玉雪}

Yuxue Wei

比亚迪建设工程有限公司西安分公司

中国·陕西 西安 710038

BYDConstruction Engineering Co., Ltd.,

Xi'an, Shaanxi, 710038, China

【摘 要】作为基础性测绘工作, 小型无人机在大比例尺地形图测绘中得到广泛应用。论 文对大比例地形图中的小型无人机实践工作进行深入分析, 以了解小型无人机在大比例 尺地形图中的实践应用价值

【Abstract】As a basic surveying and mapping work, small UAV is widely used in large-scale topographic mapping. This paper analyzes the practice of small UAV in large scale topographic map, in order to understand the practical application value of small UAV in large scale topographic map.

【关键词】比例尺;地形图; 无人机; 实践应用

【Keywords】scale; topographic map; UAV; practical application

【DOI】10.36012/se.v2i2.1552

\section{1 引言}

随着测绘技术的不断发展，小型无人机航空摄影测绘已 经广泛用于在大比例尺地形图测量实践中, 为获取大方位的 地理信息及数据提供重要的技术保障。区别于传统的 GPSRTK 全野外采集方法, 小型无人机航空摄影测绘在数据采集 效率、人工成本及绘图周期等方面具有显著优势, 同样也为小 型无人机在测绘行业的应用广度提供术支持。

\section{2 小型无人机应用特点解析}

\section{1 小型无人机结构及系统特点}

小型无人机是以航摄系统为主的无人机飞行平台，是影 像传感器为任务设备的航空遥感影像获取系统, 结合生产实 践被广泛应用于专业航拍、农业植保及设备巡检等领域,小型 无人机是由动力驱动, 其结构由螺旋桨、电子调速器、无刷电 机、电池、充电装置和遥控器组成。具有体积较小、成本低、不 易造成人员伤亡等特征, 可装配机载雷达系统、制导系统、摄 像机及传感器等设备 ${ }^{[1]}$ 。

\section{2 小型无人机数据采集及处理}

小型无人机运系统拥有小型民用无人机运行动态数据库 系统，小型无人机的飞行控制及数据采集由多核功能模块组 成,在各个功能模块之间存在明显的数据依赖关系, 向无人机 用提供天气服务于航行服务等, 对小型无人机采集的数据包 括:位置、高度、速度等运行信息等,进行实时监测。

\section{3 在测绘大比例尺地形图中的应用价值}

在传统的大比例尺地形图的测绘中, 常用到大平板仪和
小平板仪测图法及经纬仪与小平板仪联合测绘的方法, 运用 坐标法和解析法开展测绘工作, 在测绘过程中通过人工记录、 手工操作、人工绘制和人工测量坐标位置及距离面积等, 存在 劳动前度大、精准率低、绘图周期长等弊端,随着计算机技术 的迅速发展, 在现代航空摄影测绘的发展过程中,利用小型无 人机逐步替代传统测绘方式 ${ }^{[2]}$ 。

\section{3 小型无人机在大比例尺地形图中应用} 实践

\section{1 无人机像控点布置方案}

根据需要测绘地形和无人机技术设计要求开展航线设计 和航摄区域的划分, 制定相关的数据采集方法, 对测区地形条 件资料进行收集分析，运用无人机行业摄影测量数据采集回 来的数据进行处理。在像控点布置方案中, 根据 10 15 条基 本线进行布置, 在像片控制点布设中根据地形区域平面高成 设 1:1000、1:2000 的区域网布点, 平面航向基线跨度小于 7 条基线,旁向相邻跨度小于 5 条航线, 高程航向基线跨度 小于 4 条基线, 旁向相邻跨度小于 3 条航线的布点设计方案, 避免测绘段衔接区域存在漏洞。

\section{2 无人机航空摄像资料分析}

在使用无人机开展大比例尺地形图测绘时，使用平均航 摄比例尺为 $1: 23533$, 地面高度为 1500 , 摄影成图要求在 1:2000 条件下进行,通常情况下航线的间隔和旁向重叠角度 在 30\% 40\%。航向重叠角度在 60\% 70\%, 避免存在摄像漏 洞, 航向超出测绘范围的 2 5 条基线范围, 影像照片的倾斜 角度 $<4^{\circ}$, 旋偏角度 $<8^{\circ}$, 开展自东向西或从南向北的飞行质量 
标准要求, 无人机航空摄像多选在无云影遮盖的晴好天气下 进行, 从而获取较为清晰的航摄影像资料[3]。

\section{3 像控点及高程测绘}

对于大比例尺地形图测绘工作，通常采用全野外航测成 图方式进行，对于不同地貌的数据采集方式及精度规范有所 区别,对于地形图的基本高程应符合表 1 中的具体规定,利用 全站仪等设备实现像控点坐标测绘工作，根据测绘需求和测 绘进度, 利用常规像控点施测规范开展测绘, 使用双频 GPS 接 收机对控制点位进行收集，在测量像控点测绘工作完成之后 保存至参考站中, 以提升相册点测量结果的准确性和可靠性。

表 1 地形图基本等高距

\begin{tabular}{c|c|c|c}
\hline \multirow{2}{*}{ 地形类别 } & \multicolumn{3}{|c}{ 成图比例尺 } \\
\cline { 2 - 4 } & $1: 500$ & $1: 1000$ & $1: 2000$ \\
\hline 平地 & 0.5 & $0.5(1.0)$ & $1.0(0.5)$ \\
\hline 丘陵地 & $1.0(0.5)$ & 1 & 1 \\
\hline 山地 & 1 & 1 & $2.0(2.5)$ \\
\hline 高山地 & 1 & 2 & $2.0(2.5)$ \\
\hline
\end{tabular}

根据成图比例尺及实际测绘设计需要, 对于高程注记点 相对于邻近控制点的高程中误差不大于 $\pm 0.15 \mathrm{~m}$, 同时在裸露
区域和特殊地理位置使用三角高程作业方式从而实现对高程 标记位置的测量工作, 检查过程还要满足地形图检查和验收 数量的分布密度。

\section{4 结语}

通过对无人机摄影测绘技术的了解，利用小型无人机开 展大比例尺地形图测绘可有效实现高效的工作效率，随着现 代飞行平台技术的不断革新，在数据收集处理和摄像系统效 果上发挥测绘领域的巨大作用，在低人工成本上有效缩短测 绘工期，在空域管控许可的前提下开展大比例尺地形图测绘 优选小型无人机模式。

\section{参考文献}

[1]杨必胜,李健平.轻小型低成本无人机激光扫描系统研制与实 践[J].武汉大学学报(信息科学版),2018,43(12):1972-1978.

[2]柏春岗.基于小型无人机的摄影测量实践教学探讨 $[\mathrm{J}]$.北京测 绘,2018,32(9):1112-1116.

[3]黄海峰,林海玉,吕奕铭,等.基于小型无人机遥感的单体地质灾 害应急调查方法与实践[J].工程地质学报,2017,25(2):447-454.

\section{（上接第 37 页）}

\section{3 二次定向结果}

此次航测内业生产过程中, 在新工程中打开旧工程采集 的矢量文件 123.txt, 从套合结果来说基本完成。依据空中三角 测量相关规范进行检查, 发现内业加密点以及地物点对周围 野外控制点的平面中误差值为 0.2 ; 内业加密点以及高程注记 点等误差值为 0.26 , 能够达到生产要求, 说明二次定向成功 ${ }^{[2]}$ 。

\section{4 航测内业生产中二次定向应用质量把 控要点}

\section{1 做好空中三角测量的把控}

航测内业生产作业的开展,应用二次定向,其应用效果的 把控, 要保证空中三角测量的质量。在具体工作中, 要从以下 方面做好把控: (1)资料准备。开展空中三角测量作业,要准备 影像数据以及相机参数文件等。(2)建立空三区域网。在进行构 建时, 要做好影像信息以及各项限差等的核实工作, 保证数据 信息的准确性和真实性。3加密分区大小。结合测区的实际情 况、航摄分区和具体方向, 在划分加密测区时, 要尽量避开大 面积水域。(4)测区划分以及自动匹配。结合测区的实际情况, 按照匹配需求, 将测区分为多个子区, 做相应的处理。

开展空中三角测量作业, 可以采取的方法如下: (1)模拟空中 三角测量法; 2)解析空中三角测量法; 3)全数字空中三角测量
法。在具体操作的过程中,要合理选择,保证测量作业的质量。

\section{2 做好二次定向环节的把控}

在具体工作中, 要做好以下要点的把控: 1)执行二次定向 流程。严格按照操作流程,保证二次定向工作的质量。2)保持 原相对定向结果的完整性。具体操作的过程中, 对于需要进行 二次定向的工程,无论测图是否顺利完成,都需要对工程文件 进行备份处理。由于在模型中, 添加控制点或者像点等, 极易 影响相对定向结果的准确性,因此需要做好全面的把控。(3)不 可以重新定义核线范围并且采集核线。完成第一次定向后,进 行核线范围以及采集模型核线定义,接着开展测图。在开展二 次定向前, 不可以重新定义核线范围并且采集核线。

\section{5 结语}

综上所述,在航测内业生产中, 应用二次定向, 开展立体 测图工作, 能够有效解决生产问题。不仅可以保证内业生产的 进度, 还能够保证立体测图的精准度, 具有推广应用价值。

\section{参考文献}

[1]付妍. 航测内业生产中数据融合技术初探[J].科研,2016(33): $56-57$.

[2]刘俊. 航测内业生产中数据融合技术初探 $[J]$. 建筑工程技术与 设计,2015(22):62. 